\title{
Murine Gbp1 and Gbp2 are ubiquitinated independent of Toxoplasma gondii infection
}

Vesela Encheva ${ }^{1}$, Clémence Foltz ${ }^{2}$, Ambrosius P. Snijders $^{1 *}$ and Eva-Maria Frickel $2^{2^{*}}$ (D)

\begin{abstract}
Objective: The intracellular parasite Toxoplasma gondii can invade any nucleated cell residing inside a parasitophorous vacuole (PV). Upon infection, the cytokine interferon gamma (IFNY) is produced and elicits host defence mechanisms able to recognise the PV and destroy the parasite. Hereby, Guanylate binding proteins, ubiquitin and the E3 ubiquitin ligases Tripartite Motif Containing 21 (TRIM21) and TNF receptor associated factor 6 are targeted to the murine PV leading to its destruction. This study is the side product of research aiming to identify ubiquitinated substrates in a TRIM21-dependent fashion in murine cells infected with Toxoplasma.

Results: We infected IFNY-stimulated murine embryonic fibroblasts (MEFs) from either C57BL/6×129 wild-type (WT) mice or C57BL/6 TRIM21 $1^{-1-}$ mice with Toxoplasma. Using mass spectrometry, we analysed proteins in both cell backgrounds presenting with the di-glycine remnant of ubiquitination. In addition, we compared peptide levels between WT and TRIM21 ${ }^{-/-}$cells. In line with earlier reports, Gbp1 was expressed to higher levels in the C57BL/6×129 WT MEFs compared to the C57BL/6-only background TRIM21 $1^{-1-}$ MEFs. Protein expression differences in these different murine backgrounds thus precluded identification of TRIM21-dependent ubiquitinated substrates. Nevertheless, we identified and confirmed Gbp1 and Gbp2 as being ubiquitinated in a Toxoplasma-infection independent manner.
\end{abstract}

Keywords: Toxoplasma gondii, Guanylate binding proteins, Ubiquitinated substrates, Ubiquitin

\section{Introduction}

Toxoplasma gondii is an apicomplexan parasite capable of infecting all warm-blooded animals. Felines are the definitive host of the parasite enabling sexual recombination, while mice present a natural intermediate host. Global human infection rates are 30\% [1]. Immunocompetent people control the infection, however, Toxoplasma can cause congenital abnormalities, ocular disease and health problems in the immunocompromised [2].

Toxoplasma invades any nucleated cell residing in a PV [3]. The cytokine IFNY is central in controlling Toxoplasma by highly upregulating host defence proteins, amongst them the Immunity Regulated GTPases (IRGs) and Gbps. Gbps are a class of 11 active large GTPases. Gbps disrupt the Toxoplasma PV membrane and expose

\footnotetext{
*Correspondence: Bram.Snijders@crick.ac.uk; Eva.Frickel@crick.ac.uk ${ }^{1}$ Mass Spectrometry and Proteomics Platform, The Francis Crick Institute, 1 Midland Road, London NW1 1AT, UK

${ }^{2}$ Host-Toxoplasma Interaction Laboratory, The Francis Crick Institute, 1 Midland Road, London NW1 1AT, UK
}

the parasite to elimination by the host $[4,5]$. Different Toxoplasma strains (e.g. type I, II and II are the North American and European strains) vary in their genomes and thus elicit divergent host responses. Gbps for example recognize type II and III PVs, but not type I PVs, a feature driven by Toxoplasma virulence factors [6].

IFN $\gamma$-dependent targeting of type II and III Toxoplasma PVs by ubiquitin in both mouse $[7,8]$ and human cells $[9,10]$ leads to Toxoplasma control. E3 ubiquitin ligases catalyse the transfer of a ubiquitin molecule from the E2 ubiquitin-conjugating enzyme onto the protein substrate [11]. A proportion of the ubiquitin around Toxoplasma PVs in murine cells is deposited there by the E3s TRIM21 and TRAF6 [7, 8]. TRIM21 is critically important for survival of Toxoplasma infection in vivo and is linked to the production of cytokines and parasite clearance, as well as the levels of K63-linked ubiquitin around the PV [8]. The ubiquitin/TRIM21/TRAF6 system targets the same vacuoles recognized by the Gbps, a process that is seeded by IRGs $[7,12]$. 
Ubiquitinated protein substrates in murine or human cells infected with Toxoplasma gondii are unknown. The consequence of ubiquitination of host defence proteins could be manifold-these proteins could participate in immune-mediated signaling or be targets of pathogen defence strategies. Knowledge of these ubiquitinated substrates could lead to novel anti-Toxoplasma therapeutic strategies in humans. Since we currently do not know of any E3 ubiquitin ligase responsible for targeting the Toxoplasma PV in human cells, research along these lines is limited to murine systems.

Here, we aimed to find ubiquitinated substrates in a TRIM21-dependent fashion in murine cells infected with Toxoplasma. The goal was to identify Toxoplasma PVresident ubiquitinated proteins that played important parts in pathogen defence. This study is the side product of our identification of TRIM21 as an essential player in murine host-mediated resistance to Toxoplasma in vivo [8]. Protein expression difference in the divergent murine backgrounds of the wild-type versus TRIM $21^{-1-}$ cells precluded identification of TRIM21-specific substrates. However, we present the single observation of analysing and defining by mass spectrometry ubiquitinated substrates in IFN $\gamma$-treated Toxoplasma-infected WT and TRIM21 ${ }^{-1-}$ MEFs. We identified and confirmed that Gbp1 and Gbp2 are ubiquitinated in WT MEFs in a Toxoplasma-infection independent fashion.

\section{Main text Methods \\ Cell culture, parasite culture and infection}

WT and TRIM21 ${ }^{-1-}$ MEFs were previously generated and cultivated in Dulbecco's Modified Eagle Medium (DMEM) with $10 \%$ fetal bovine serum (FBS) at $37^{\circ} \mathrm{C}$ and $5 \% \mathrm{CO}_{2}$ [13]. Mice used for MEFs are on a project license approved by the Home Office, UK, under the Animals Scientific Procedures Act 1986 and the procedure was approved by the local ethical committee of the Francis Crick Institute Ltd. Although the Gbp1 gene is present, following in vivo poly I:C stimulation, Gbp1 protein is not expressed in mice in a pure C57BL/6 background $[14,15]$. Thus, WT MEFs were on a C57BL/6×129 background and derived from animal bred at the Francis Crick Insitute Ltd, while TRIM21 ${ }^{-1-}$ MEFs were on a pure C57BL/6 background and purchased from the Jackson Laboratory. Toxoplasma gondii type I (RH) and type II $\mathrm{Pru}$ ) were maintained by serial passage on monolayers of human foreskin fibroblasts (HFFs) (ATCC, SCRC-1041) as described previously and were a gift from Jeroen Saeij (UC Davis, USA) [16]. A multiplicity of infection (MOI) of five was used after induction with $100 \mathrm{U} / \mathrm{mL}$ murine recombinant IFN $\gamma$ (R\&D Systems) for $16 \mathrm{~h}$. For SILAC, cells were cultured in DMEM supplemented with light or heavy L-arginine and L-lysine. Unlabelled, hydrochloride forms of $\mathrm{L}$-arginine and L-lysine (ROKO) were from Sigma-Aldrich (light isotopes). Hydrochloride forms of L-arginine [13C6, 15N4] and L-lysine [13C6, 15N2] (R10K8) were from CK Isotopes (CNLM-291-H-0.5 and CNLM-539-H-0.25). The infected monolayer of HFF was cultured in DMEM (light and heavy) supplemented with $1 \%$ dialysed FBS for six doublings. WT and TRIM $21^{-1-}$ MEFs were cultured in both light and heavy media, supplemented with $10 \%$ dialysed FBS and passaged for five doublings.

\section{Sample preparation for $L C-M S / M S$}

DiGly peptides from Toxoplasma-infected MEFs were generated as previously described [17] with full details described in Additional file 1.

\section{LC-MS/MS}

Peptides were resuspended in $0.1 \%$ TFA and loaded on $50 \mathrm{~cm}$ Easy Spray PepMap column $(75 \mu \mathrm{m}$ inner diameter, $2 \mu \mathrm{m}$ particle size, Thermo Fisher Scientific, ES803) equipped with an integrated electrospray emitter. Reverse phase chromatography was performed using the RSLC nano U3000 (Thermo Fisher Scientific) with a binary buffer system at a flow rate of $250 \mathrm{~nL} / \mathrm{min}$. Solvent A was $0.1 \%$ formic acid (Thermo Fisher, 28904, 5\% DMSO (Sigma, 41640), and solvent B was $80 \%$ acetonitrile, 0.1\% formic acid, 5\% DMSO. The diGly enriched samples were run on a linear gradient of solvent B (2-40\%) in $90 \mathrm{~min}$, total run time of 120 min including column conditioning. The nanoLC was coupled to a Q Exactive mass spectrometer using an EasySpray nano source (Thermo Fisher Scientific).

The Q Exactive was operated in data-dependent mode acquiring HCD MS/MS scans $(\mathrm{R}=17,500)$ after an MS1 scan $(R=70,000)$ on the 10 most abundant ions using MS1 target of $1 \times 10^{6}$ ions, and MS2 target of $5 \times 10^{4}$ ions. The maximum ion injection time utilised for MS2 scans was $120 \mathrm{~ms}$, the HCD normalized collision energy was set at 28, the dynamic exclusion was set at $10 \mathrm{~s}$, and the peptide match and isotope exclusion functions were enabled.

\section{Data processing and analysis}

The data were processed and analysed according to methods detailed in Additional file 1.

\section{Immunoprecipitation and immunoblot}

All steps were performed on ice. Cells were washed twice in PBS and lysed in ice cold lysis buffer $(25 \mathrm{mM}$ Tris $\mathrm{HCl}$ (Roche, 10708976001) pH 7.4, $5 \mathrm{mM} \mathrm{MgCl}_{2}$ (Sigma, M8266), $150 \mathrm{mM} \mathrm{NaCl}$ (Sigma, S7653), 0.5\% NP40 (BDH Laboratory Supplies, 56009), cOmplete EDTA-free 
protease inhibitor cocktail (Sigma, 11836170001)). Total protein lysate was equalised between samples and $0.5 \mathrm{mg}$ were immunoprecipitated with Ubiquitin 1 Tandem UBA (TUBE1) agarose (BostonBiochem, AM-125) according to the manufacturer's recommendation. Immunoprecipitated proteins were eluted from the beads at $92{ }^{\circ} \mathrm{C}$ for $10 \mathrm{~min}$ in $1 \times$ NuPAGE protein loading buffer (Thermo Fisher, NP0008) supplemented with $1 \mathrm{mM}$ DTT (Sigma, D5545). Samples were separated on a $4-12 \%$ gradient SDS-PAGE (Thermo Fisher, NP0322BOX). All of the immunoprecipitate was loaded and $5 \mu \mathrm{g}$ of the original protein lysate per sample. Proteins were transferred onto nitrocellulose membrane (GE Health care Life Sciences, 10600001) using semi-dry immunoblotter (Biorad TransBlot SD Cell). Blots were blocked at RT for $1 \mathrm{~h}$ in $5 \%$ nonfat dried milk/PBS solution and probed with anti-Gbp1 (generated by us in [6]) and anti-Gbp2 antibodies (kind gift from Joern Coers, Duke; published in [18]) in 5\% non-fat dried milk/PBS + 0.1\% Tween-20 (Sigma, P1379). Blots were then incubated with HRP-conjugated antirabbit secondary antibody (Life Technologies, G21234) in $5 \%$ non-fat dried milk/PBS $+0.1 \%$ Tween-20 (Sigma, P1379) and developed with Pierce ECL Western Blotting Solution (Thermo Scientific, 32106).

\section{Results and discussion}

The addition of the small protein ubiquitin to substrate proteins can alter the fate of these proteins and serve to send the protein to degradation or to induce cellular responses [19]. Ubiquitin as an anti-pathogen response has been widely studied, yet ubiquitinated protein substrates in murine or human cells infected with Toxoplasma gondii are unknown. Here, we aimed to identify ubiquitinated substrates in a TRIM21-dependent fashion in murine cells infected with Toxoplasma. We performed a stable isotope labelling with an amino acids (Silac) proteomics experiment in duplicate with Silac label reversal in Toxoplasma-infected murine cells. We compared IFNY-stimulated wild-type (WT, C57BL/6×129) and TRIM21 ${ }^{-1-}$ cells (C57BL/6) infected with type II Toxoplasma. Peptides originating from protein ubiquitination sites were affinity enriched using an antibody that is specific for lysine containing peptides with isolinked diglycine motifs as described elsewhere [20]. These peptides are generated after trypsin digestion of the cell lysate containing E3 ligase substrates. In comparing C57BL/6×129 WT MEFs with pure C57BL/6 TRIM21 $1^{-I-}$ MEFs, we observed the predicted expression difference of Gbp1 protein between these divergent murine backgrounds. Cells from 129 mice had previously been classed as "responder" cells in terms of Gbp1 expression versus $\mathrm{C} 57 \mathrm{BL} / 6$ belonging to the group of Gbp1 nonexpressing and thus termed "non-responder" cells [14].
This distinction had been made on the basis of in vivo poly I:C stimulated mice [14]. Nevertheless, another report does find transcriptional upregulation of $G b p 1$ after IFN $\gamma$ stimulation of ANA-1 macrophages, derived from C57BL/6J mice [21]. The observed difference to our proteomics data (derived from IFN $\gamma$-stimulated MEFs) could be a cell-type dependent variation of Gbp1 expression (MEFs versus macrophages) or it is possible that the Gbp1 transcripts that are observed in the ANA-1 macrophages do not translate into protein. Regardless, it is interesting to note that Gbp1 does exhibit expression differences in divergent inbred murine strains, while Gbp2 is more homogenously expressed between these murine backgrounds. The divergent protein expression of Gbp1 that was observed between the different murine background MEFs however also precluded our robust analysis if any of the ubiquitinated substrates were indeed TRIM21-dependent.

Surprisingly, unmodified and di-Gly modified peptides derived from Gbp1 to Gbp2 demonstrated that both of these GTPases were themselves ubiquitinated in Toxoplasma-infected cells (Fig. 1, Additional file 2: Table S1). This is in contrast to Traver et al. who have shown that IRGs, but not Gbps, can be immunoprecipitated with a TUBE1 resin that specifically binds Lys63-linked ubiquitin [18]. For Gbp1 22 di-Gly modified peptides were identified which corresponded to 10 distinct ubiquitination sites (Fig. 2). For Gbp2 there were 36 di-Gly peptides and total of 25 ubiquitination sites (Fig. 2). The majority of the ubiquitination sites identified were localized in the C-terminal part of the proteins (Fig. 2). Very few ubiquitination sites on Gbp1 and Gbp2 have been previously described in the literature. The only two known sites, which we also detected in this study, are K232 and K389 on Gbp2 (https://www.phosphosite.org).

Additionally, several other IFN $\gamma$-inducible GTPases were found to be ubiquitinated. These include four sites on Igtp1, 10 sited on Irgm1, 12 sites on Irgm2, and 15 on Gvin1 (Additional File 2: Table S1).

In order to confirm that Gbp1 and Gbp2 are ubiquitinated, we analysed C57BL/6×129 MEFs stimulated with IFNY and infected with Toxoplasma type I and II by immunoblot. We performed immunoprecipitation of ubiquitinated endogenous proteins followed by immunoblotting for Gbp1 and Gbp2. We detected both Gbps as being ubiquitinated (Fig. 3). Interestingly, Gbp1 and Gbp2 are both ubiquitinated regardless of cellular Toxoplasma infection status. Ubiquitination of Gbps has recently garnered attention in serving as a pathogenmediated defence mechanism against these GTPases. A Shigella flexneri-encoded E3 ubiquitin ligase can target human GBP1 for ubiquitination and subsequent degradation, thus neutralising its original function of inhibiting 

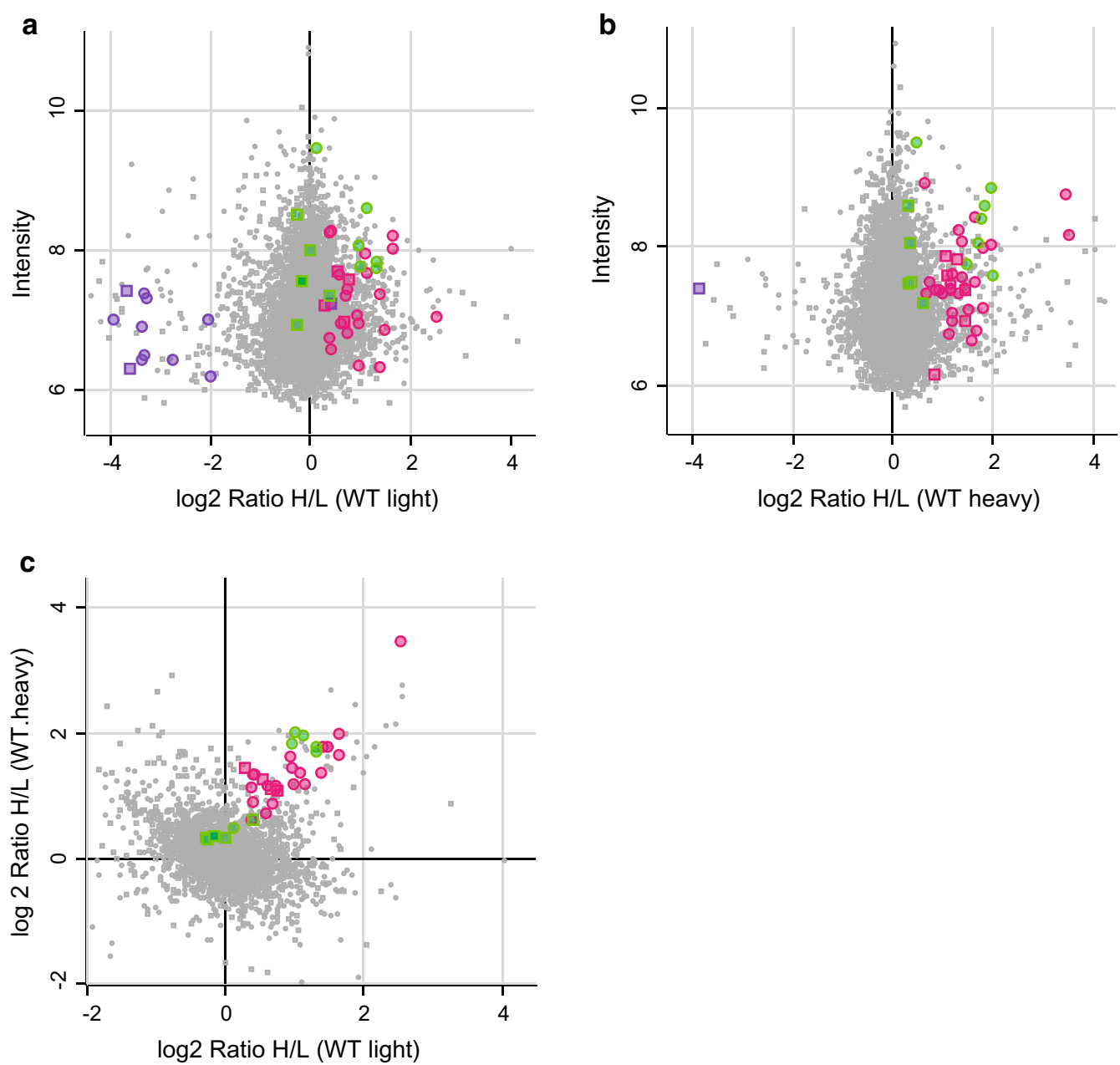

Fig. 1 Mass spectrometry reveals Gbp1 and Gbp2 are ubiquitinated during Toxoplasma infection. a, b Ratio intensity plot and c correlation plot of peptide levels recovered by immunoprecipitation with a di-Gly antibody after trypsin digestion of IFNY-stimulated C57BL/6×129 TRIM21 KO and wild-type C57BL/6×129 MEFs infected with Toxoplasma. Gbp1 and Gbp2 associated peptides both recovered as diGly-modified and- unmodified are marked. Gbp1 peptides are in purple and Gbp2 peptides are pink. Shared peptides are in green. Circles represent di-Gly modified peptides and squares are unmodified peptides

actin-based bacterial mobility [22-24]. In our case, as the ubiquitination of Gbp1 and Gbp2 are Toxoplasma-infection independent, it remains to be investigated whether non-ubiquitinated versus ubiquitinated Gbp1 and Gbp2 would exhibit different localisation in the cell. It is conceivable that global Gbp1 and Gbp2 ubiquitination status might not change during infection, while this modification might target the defence proteins specifically to Toxoplasma PVs to excert their function. 
>sp|Q01514|GBP1_MOUSE Guanylate-binding protein 1 OS=Mus musculus GN=Gbp1 PE=1

$\mathrm{SV}=1$

MASEIHMSEPMCLIENTEAQLVINQEALRILSAITQPVVVVAIVGLYRTGKSYLMNKLAG

KRTGFSLGSTVQSHTKGIWMWCVPHPKKAGQTLVLLDTEGLEDVEKGDNQNDCWIFALAV

LLSSTFIYNSIGTINQQAMDQLHYVTELTDLIKSKSSPDQSDVDNSANFVGFFPIFVWTL

RDFSLDLEFDGESITPDEYLETSLALRKGTDENTKKFNMPRLCIRKFFPKRKCFIFDRPG

DRKQLSKLEWIQEDQLNKEFVEQVAEFTSYIFSYSGVKTLSGGITVNGPRLKSLVQTYVS

AICSGELPCMENAVLTLAQIENSAAVQKAITYYEEQMNQKIHMPTETLQELLDLHRTCER

EAIEVFMKNSFKDVDQKFQEELGAQLEAKRDAFVKKNMDMSSAHCSDLLEGLFAHLEEEV

KQGTFYKPGGYYLFLQRKQELEKKYIQTPGKGLQAEVMLRKYFESKEDLADTLLKMDQSL

TEKEKQIEMERIKAEAAEAANRALAEMQKKHEMLMEQKEQSYQEHMKQLTEKMEQERKEL

MAEQQRIISLKLQEQERLLKQGFQNESLQLRQEIEKIKNMPPPRSCTIL

$>$ sp $\mid$ Q9ZOE6 $\mid$ GBP2_MOUSE Guanylate-binding protein 2 OS=Mus musculus GN=Gbp2 PE=1

$\mathrm{SV}=1$

MASEIHMSEPMCLIENTEAQLVINQEALRILSAITQPVVVVAIVGLYRTGKSYLMNKLAG

KRTGFSLGSTVQSHTKGIWMWCVPHPKKAGQTLVLLDTEGLEDVEKGDNQNDCWIFALAV

LLSSTFIYNSIGTINQQAMDQLHYVTELTDLIKSKSSPDQSGVDDSANFVGFFPTFVWTL

RDFSLELEVNGKPVTSDEYLEHSLTLKKGADKKTKSFNEPRLCIRKFFPKRKCFIFDRPA

QRKQLSKLETLREEELCGEFVEQVAEFTSYILSYSSVKTLCGGIIVNGPRLKSLVQTYVG

AISNGSLPCMESAVLTLAQIENSAAVQKAITHYEEQMNQKIQMPTETLQELLDLHRPIES

EAIEVFLKNSFKDVDQKFQTELGNLLVAKRDAFIKKNMDVSSARCSDLLEDIFGPLEEEV

KLGTFSKPGGYYLFLQMRQELEKKYNQAPGKGLQAEAMLKNYFDSKADVVETLLQTDQSL

TEAAKEVEEERTKAEAAEAANRELEKKOKEFELMMQQKEKSYQEHVKKLTEKMKDEQKQL

LAEQENIIAAKLREQEKFLKEGFENESKKLIREIDTLKQNKSSGKCTIL

Fig. 2 Identified ubiquitinated lysines of Gbp1 and Gbp2. FASTA sequence of Gbp1 and Gbp2 with the localisation of ubiquitinated lysines identified in this study marked. A total of 10 ubiquitinated lysines were found on Gbp1 including K51, K57, K87, K372, K389, K443, K444, K493, K532 and K560. Gbp2 had 25 modified lysines including K51, K57, K87, K215, K232, K372, K389, K396, K444, K451, K460, K509, K518, K520, K532, K534, K538, K551, K557, K560, K568, K569, K578, K581 and K585. Modified lysines found on peptides with identical sequences between Gbp1 and Gbp2 (shared peptides) are colored in green. Unique sites are colored in purple for Gbp1 and pink for Gbp2
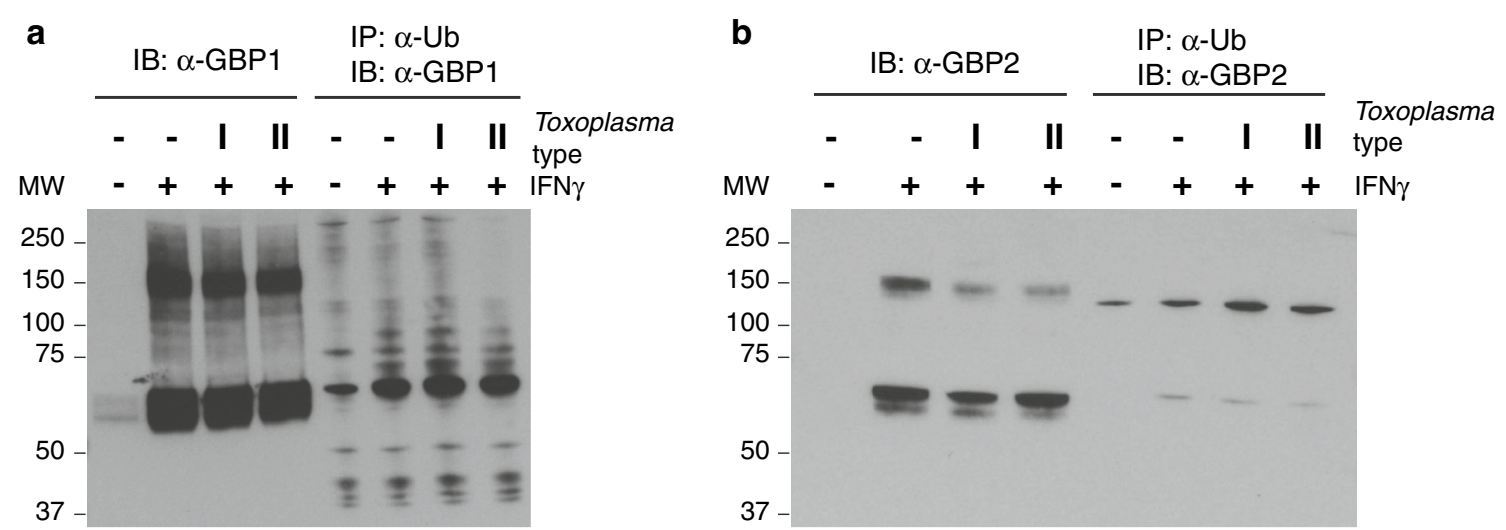

Fig. 3 Confirmation of Gbp1 and Gbp2 ubiquitination by ubiquitin immunoprecipitation. $\mathbf{a}$, b Immunoprecipitation using TUBE2 resin of ubiquitinated Gbp1 and Gbp2 proteins in IFNY- or non-stimulated naïve or infected WT C57BL/6×129 MEFs. Lysate: 5 Mg of the input. Data representative of three independent experiments. MW molecular weight ( $\mathrm{kDa})$ 


\section{Limitations}

As we are comparing MEFs from divergent murine backgrounds, we could not infer TRIM21-specific substrates from our experiments.

\section{Additional files}

\section{Additional file 1. Additional Methods.}

Additional file 2: Table S1. List of all ubiquitinated protein sites identified in Toxoplasma-infected MEFs. Maxquant processed data taken from the modifiedpeptides.txt output table. Log2 normalised ratios for the indicated peptide sequences are displayed.

\section{Abbreviations}

Gbp: guanylate binding protein; IFNg: interferon gamma; MEFs: murine embryonic fibroblasts; PV: parasitophorous vacuole; TRAF6: TNF receptor associated factor 6; TRIM21: Tripartite Motif Containing 21; WT: wild-type FBS: fetal bovine serum; DMEM: Dulbecco's Modified Eagle Medium; RH: Toxoplasma type I; Pru: Toxoplasma type II; MOI: multiplicity of infection; HFF: human foreskin fibroblasts; DTT: dithiothreitol; IAP: immunoaffinity purification; IRG: immunity related GTPases; RT: room temperature; TUBE1: Ubiquitin 1 Tandem UBA.
\end{abstract}

\section{Authors' contributions}

$V E, C F, A P S$ and EMF designed experiments and wrote the manuscript. CF, VE and EMF conducted experiments, APS and EMF supervised the study. All authors read and approved the final manuscript.

\section{Acknowledgements}

We would like to thank Joern Coers for the anti-Gbp2 antibody.

\section{Competing interests}

The authors declare that they have no competing interests.

\section{Availability of data and materials}

The mass spectrometry proteomics datasets generated during the current study have been deposited to and are available at the ProteomeXchange Consortium [25] via the PRIDE partner repository with the dataset identifier PXD003217.

\section{Consent for publication}

Not applicable.

\section{Ethics approval and consent to participate}

All procedures involving mice for the generation of MEFs were approved by the local ethical committee of the Francis Crick Institute Ltd, Mill Hill Laboratory and are part of a project license approved by the Home Office, UK, under the Animals Scientific Procedures Act 1986

\section{Funding}

This work was supported by the Francis Crick Institute, which receives its core funding from Cancer Research UK (FC001076/FC001999), the UK Medical Research Council (FC001076/FC001999), and the Wellcome Trust (FC001076/ FC001999). Eva-Maria Frickel was supported by a Wellcome Trust Career Development Fellowship (091664/B/10/Z). Clémence Foltz was the recipient of a Boehringer Ingelheim Fonds Ph. D. Fellowship and a Medical Research Council Studentship (MC_ST_U11033). The funders had no role in the design of the study.

\section{Publisher's Note}

Springer Nature remains neutral with regard to jurisdictional claims in published maps and institutional affiliations.
Received: 23 October 2017 Accepted: 24 February 2018

Published online: 06 March 2018

\section{References}

1. Montoya JG, Liesenfeld O. Toxoplasmosis. Lancet. 2004;363:1965-76.

2. Muñoz M, Liesenfeld O, Heimesaat MM. Immunology of Toxoplasma gondii. Immunol Rev. 2011;240:269-85.

3. Lingelbach $\mathrm{K}$, Joiner KA. The parasitophorous vacuole membrane surrounding Plasmodium and Toxoplasma: an unusual compartment in infected cells. J Cell Sci. 1998;111(Pt 11):1467-75.

4. Yamamoto M, Okuyama M, Ma JS, Kimura T, Kamiyama N, Saiga H, et al. A Cluster of interferon- $\gamma$-inducible p65 GTPases plays a critical role in host defense against Toxoplasma gondii. Immunity. 2012;37:302-13.

5. Selleck EM, Fentress SJ, Beatty WL, Degrandi D, Pfeffer K, Virgin HW, et al. Guanylate-binding protein 1 (Gbp1) contributes to cell-autonomous immunity against Toxoplasma gondii. PLoS Pathog. 2013;9:e1003320.

6. Virreira Winter S, Niedelman W, Jensen KD, Rosowski EE, Julien L, Spooner $\mathrm{E}$, et al. Determinants of GBP recruitment to Toxoplasma gondii vacuoles and the parasitic factors that control it. PLoS ONE. 2011;6:e24434.

7. Haldar AK, Foltz C, Finethy R, Piro AS, Feeley EM, Pilla-Moffett DM, et al. Ubiquitin systems mark pathogen-containing vacuoles as targets for host defense by guanylate binding proteins. Proc Natl Acad Sci USA. 2015;112:E5628-37.

8. Foltz C, Napolitano A, Khan R, Clough B, Hirst EM, Frickel EM. TRIM21 is critical for survival of Toxoplasma gondii infection and localises to GBPpositive parasite vacuoles. Sci Rep. 2017;7:5209.

9. Clough B, Wright JD, Pereira PM, Hirst EM, Johnston AC, Henriques R, et al. K63-linked ubiquitination targets Toxoplasma gondii for endolysosomal destruction in IFNY-stimulated human cells. PLoS Pathog. 2016;12:e1006027.

10. Selleck EM, Orchard RC, Lassen KG, Beatty WL, Xavier RJ, Levine B, et al. A noncanonical autophagy pathway restricts Toxoplasma gondii growth in a strain-specific manner in IFN- $\gamma$-activated human cells. MBio. 2015;6:e01157-2015.

11. Pickart CM. Ubiquitin enters the new millennium. Mol Cell. 2001;8:499-504.

12. Lee $Y$, Sasai M, Ma JS, Sakaguchi N, Ohshima J, Bando H, et al. p62 plays a specific role in interferon- $\gamma$-induced presentation of a Toxoplasma vacuolar antigen. Cell Rep. 2015;13:223-33.

13. Yoshimi R, Chang T-H, Wang H, Atsumi T, Morse HC, Ozato K. Gene disruption study reveals a nonredundant role for TRIM21/Ro52 in NF-kappaB-dependent cytokine expression in fibroblasts. J Immunol. 2009;182:7527-38.

14. Staeheli P, prochazka M, Steigmeier PA, Haller O. Genetic control of interferon action: mouse strain distribution and inheritance of an induced protein with guanylate-binding property. Virology. 1984;137:135-42.

15. Cheng YS, Patterson CE, Staeheli P. Interferon-induced guanylate-binding proteins lack an N(T)KXD consensus motif and bind GMP in addition to GDP and GTP. Mol Cell Biol. 1991:11:4717-25.

16. Garnham PC, Baker JR, Bird RG. Fine structure of cystic form of Toxoplasma gondii. Br Med J. 1962;1:83-4.

17. Boeing S, Wiliamson L, Encheva V, Gori I, Saunders RE, Instrell R, et al. Multiomic analysis of the UV-induced DNA damage response. Cell Rep. 2016;15:1597-610.

18. Traver MK, Henry SC, Cantillana V, Oliver T, Hunn JP, Howard JC, et al. Immunity-related GTPase M (IRGM) proteins influence the localization of guanylate-binding protein 2 (GBP2) by modulating macroautophagy. J Biol Chem. 2011;286:30471-80.

19. Akutsu M, Dikic I, Bremm A. Ubiquitin chain diversity at a glance. J Cell Sci. 2016:129:875-80.

20. Udeshi ND, Svinkina T, Mertins P, Kuhn E, Mani DR, Qiao JW, et al. Refined preparation and use of anti-diglycine remnant (K- $\varepsilon-\mathrm{GG}$ ) antibody enables routine quantification of $10,000 \mathrm{~s}$ of ubiquitination sites in single proteomics experiments. Mol Cell Proteom. 2013;12:825-31.

21. Degrandi D, Konermann C, Beuter-Gunia C, Kresse A, Würthner J, Kurig $\mathrm{S}$, et al. Extensive characterization of IFN-induced GTPases mGBP1 to mGBP10 involved in host defense. J Immunol. 2007;179:7729-40. 
22. Li P, Jiang W, Yu Q, Liu W, Zhou P, Li J, et al. Ubiquitination and degradation of GBPs by a Shigella effector to supress host defence. Nature. 2017;551:378-83.

23. Wandel MP, Pathe C, Werner El, Ellison CJ, Boyle KB, von der Malsburg A, et al. GBPs inhibit motility of Shigella flexneri but are targeted for degradation by the bacterial ubiquitin ligase IpaH9.8. Cell Host Microbe. 2017;22:507-18.
24. Piro AS, Hernandez D, Luoma S, Feeley EM, Finethy R, Yirga A, et al. Detection of cytosolic Shigella flexneri via a C-terminal triple-arginine motif of GBP1 inhibits actin-based motility. mBio. 2017:8:e1979-2017.

25. Vizcaíno JA, Deutsch EW, Wang R, Csordas A, Reisinger F, Ríos D, et al. ProteomeXchange provides globally coordinated proteomics data submission and dissemination. Nat Biotechnol. 2014;32:223-6.

\section{Submit your next manuscript to BioMed Central and we will help you at every step:}

- We accept pre-submission inquiries

- Our selector tool helps you to find the most relevant journal

- We provide round the clock customer support

- Convenient online submission

- Thorough peer review

- Inclusion in PubMed and all major indexing services

- Maximum visibility for your research

Submit your manuscript at

www.biomedcentral.com/submit 